\title{
Magnetic Diversity in Heteroisocorroles: Aromatic Pathways in 10-Heteroatom-Substituted Isocorroles
}

\author{
Cina Foroutan-Nejad* ${ }^{\dagger} \dagger$ and Abhik Ghosh*,\$0
}

${ }^{\dagger}$ CEITEC - Central European Institute of Technology, Masaryk University, Kamenice 5, CZ-62500 Brno,

Czech Republic

${ }^{\ddagger}$ Department of Chemistry and Center for Theoretical and Computational Chemistry, UiT - The Arctic University of Norway, 9037 Tromsø, Norway

\section{Supporting Information}

ABSTRACT: A recent study on magnetically induced currents in 10-isocorrole derivatives indicated that both the free-base and metal-complexed forms of the unsubstituted macrocycle are homoaromatic. Furthermore, depending on the substituents at the 10-position, the aromatic character was found to swing between substantially homoaromatic to substantially antihomoaromatic. Heteroisocorroles, in which the saturated 10-position has been replaced by a heteroatom-containing group $\mathrm{X}$, are predicted to exhibit even more dramatic variations in aromatic character, ranging from strongly aromatic $(X=O, N H$, $\mathrm{PH}$, and $\mathrm{S})$ to strongly antiaromatic $(\mathrm{X}=\mathrm{BH}$ and $\mathrm{CO})$. Interestingly, the experimentally studied $\mathrm{X}=\mathrm{SiMe}_{2}$ case does not appear to sustain a significant global ring current.

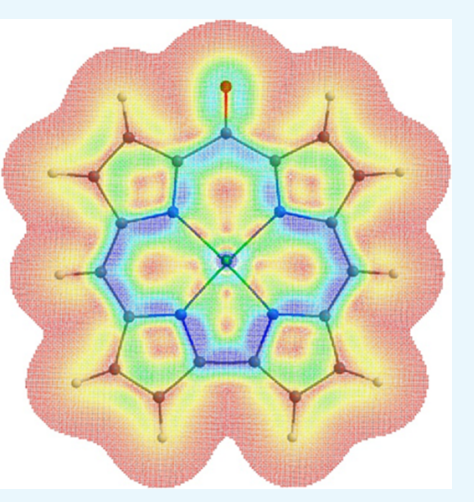

\section{INTRODUCTION}

Isocorroles are a class of hybrid ligands that combine the diprotic character of porphyrins and the contracted $\mathrm{N}_{4}$ cavity of corroles. ${ }^{1-4}$ Heteroisocorroles (a term that we prefer over heterocorroles), in which the saturated meso carbon of isocorroles is substituted by a heteroatom, vastly expand the scope of these ligands. ${ }^{5,6}$ Like other sterically constrained porphyrinoids, such as porphyrazines ${ }^{7}$ and corroles, ${ }^{8} 10$-heteroisocorroles have been shown to stabilize unusual transition metal spin states, such as intermediate-spin $\mathrm{Fe}$ (III), ${ }^{9,10}$ whereas strong absorption in the red and near-infrared (IR) range may result in applications as near-IR dyes and in photodynamic therapy. ${ }^{11}$ Additionally, Bröring and co-workers have noted fascinating variations of

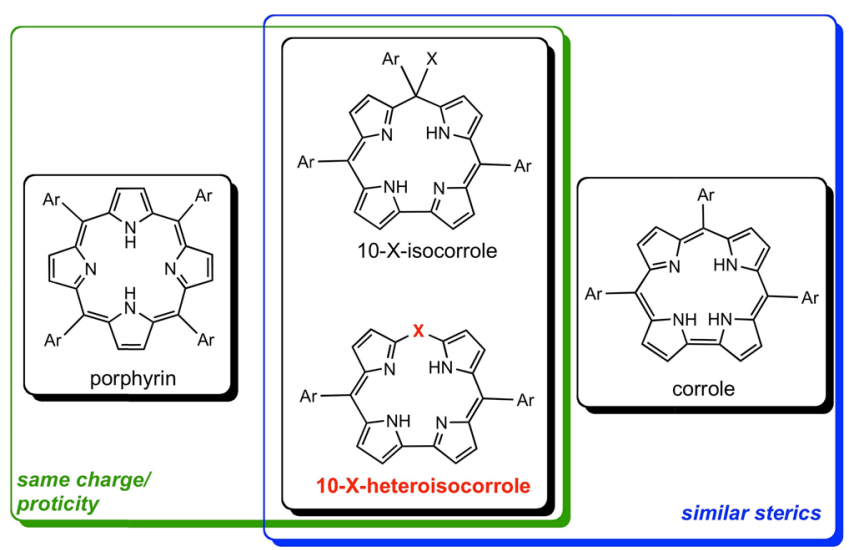

Figure 1. Structural relationships among free-base porphyrin, corrole, isocorrole, and heteroisocorrole.

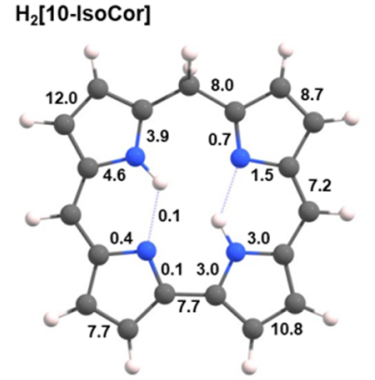

(a)

Ni[10-IsoCor]

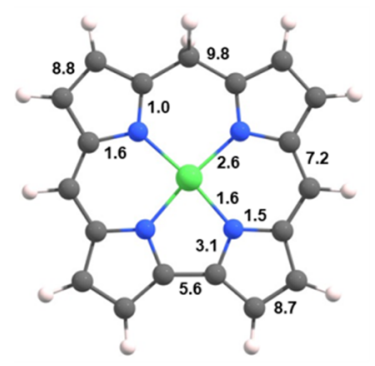

(c)

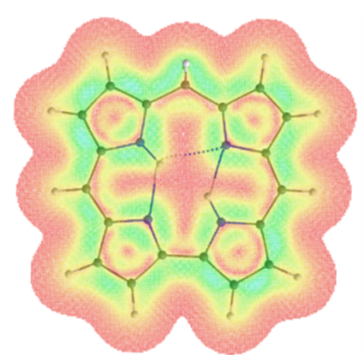

(b)

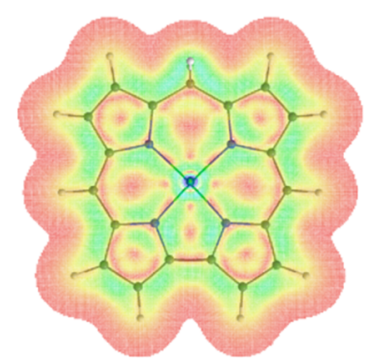

(d)
Figure 2. Integrated currents $(\mathrm{nA} / \mathrm{T}$, left $)$ and current density plots (right) for $\mathrm{H}_{2}$ [10-IsoCor] and $\mathrm{Ni}$ [10-IsoCor]. Note: Throughout this paper, the current density has been plotted $1 \mathrm{~b}$ above the mean molecular plane, and the colors blue $(0.001 \mathrm{au})$ to red $(0.000 \mathrm{au})$ represent strong to weak current densities.

Received: October 2, 2018

Accepted: November 8, 2018

Published: November 21, 2018 

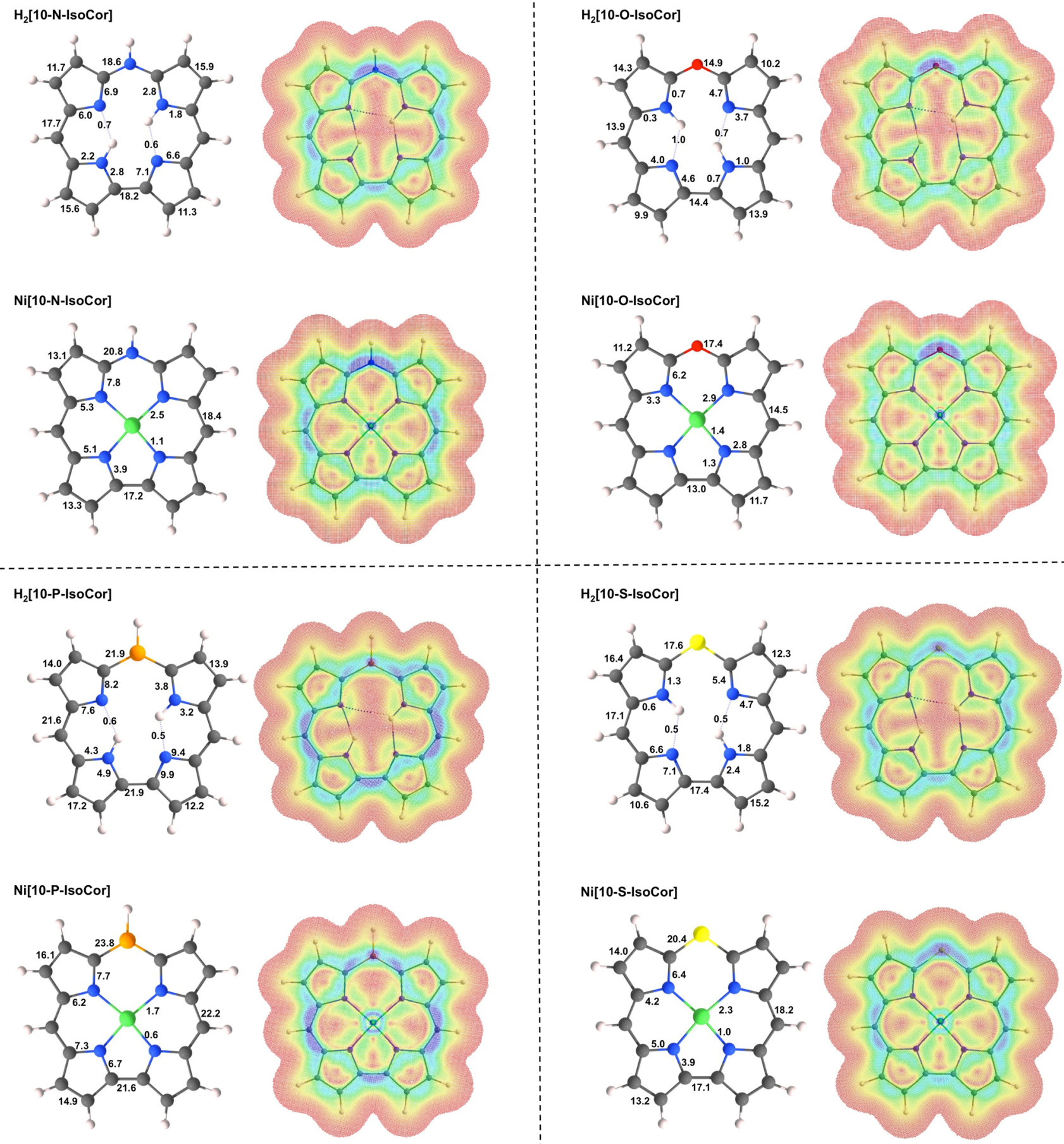

$\mathrm{Ni}[10-\mathrm{S}-$ IsoCor]

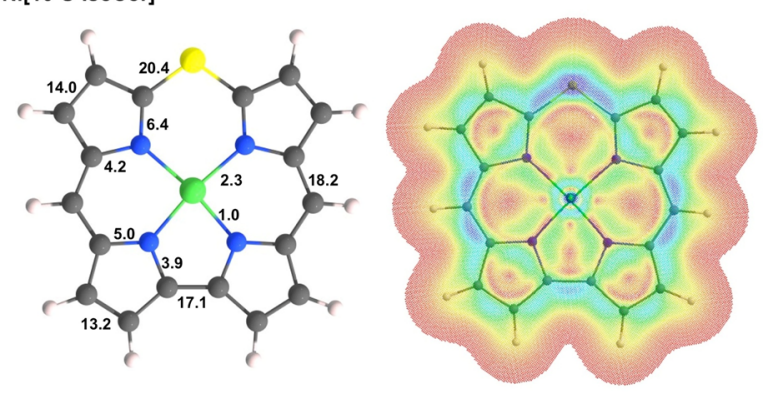

Figure 3. Integrated currents $(\mathrm{nA} / \mathrm{T})$ and current density plots for aromatic heteroisocorrole derivatives.

aromatic character among 10-heteroisocorrole derivatives, characterizing the observations as "a welcome subject for future in-depth theoretical studies". 5 On a related note, recent density functional theory (DFT) calculations of magnetically induced current densities have shown that the parent isocorrole is homoaromatic (i.e., aromatic in spite of a formally interrupted conjugation) and the aromatic character is susceptible to dramatic substituent effects. ${ }^{12}$ Herein, we have extended analogous ${\mathrm{B} 3 L Y \mathrm{~L}^{13-15} / \text { def2-TZVP }}^{16}$ current density analyses ${ }^{17-22}$ to free-base and $\mathrm{Ni}(\mathrm{II})$-complexed heteroisocorroles (Figure 1) (see Methods). In terms of their current density distribution, the compounds have been found to be tremendously diverse, ranging from strongly aromatic to strongly antiaromatic, somewhat reminiscent of the variations in the aromatic character of the homotropylium cation as a function of out-of-plane deformation. ${ }^{23,24}$ Consistent with predictions by Breslow, ${ }^{25}$ who incidentally coined the term 'antiaromatic', ${ }^{26}$ antiaromatic porphyrinoids have recently found application as components of highly conducting molecular circuits. ${ }^{27}$ A host of other fascinating spectroscopic signatures has also been attributed to antiaromatic porphyrinoids. ${ }^{28}$ The identification of new antiaromatic heteroisocorroles, therefore, 
$H_{2}[10-B-I s o C o r]$
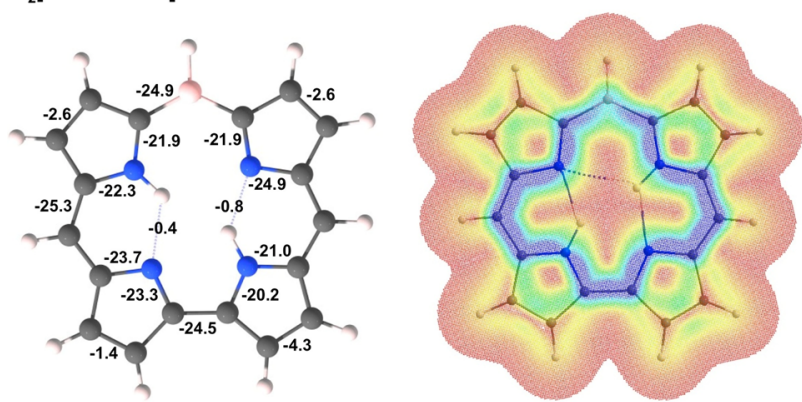

Ni[10-B-IsoCor]
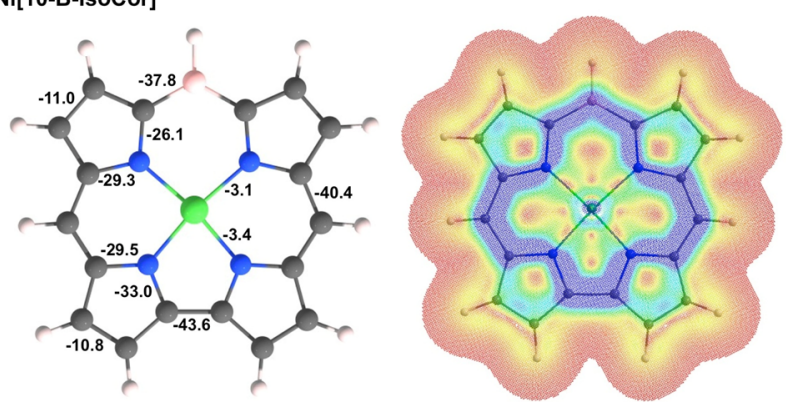
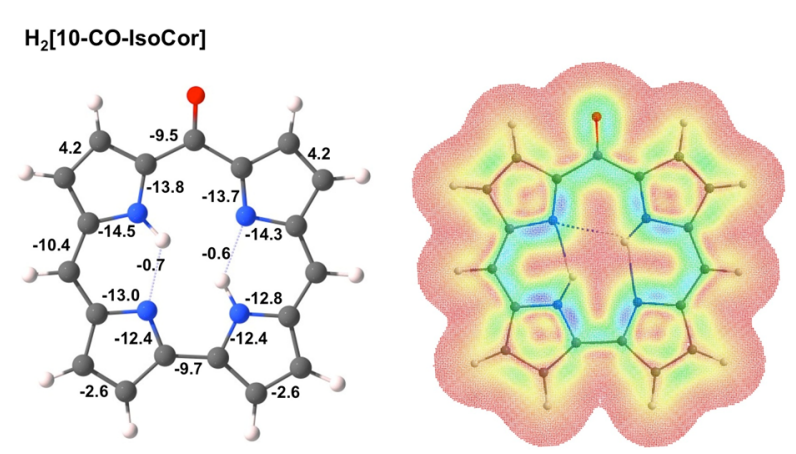

Ni[10-CO-IsoCor]
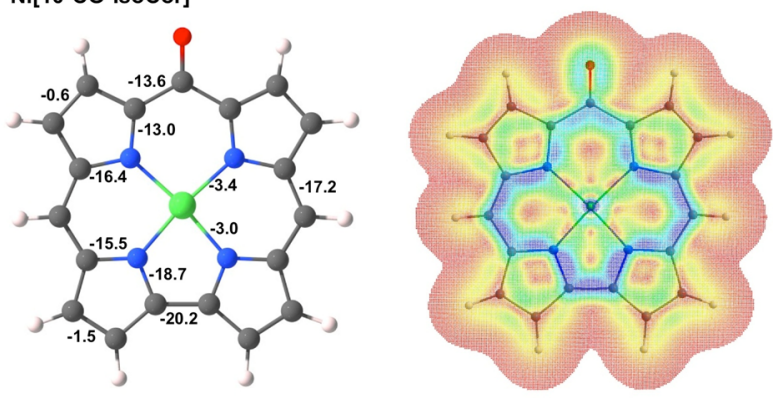

Figure 4. Integrated currents $(\mathrm{nA} / \mathrm{T})$ and current density plots for antiaromatic heteroisocorrole derivatives.

in our view, is a particularly intriguing aspect of the present study.

\section{RESULTS AND DISCUSSION}

The unsubstituted isocorroles $\mathrm{H}_{2}$ [10-IsoCor] and $\mathrm{Ni}$ [10IsoCor] sustain moderate diatropic currents of 5.6-9.8 nA/T along the $\mathrm{C}_{\alpha}-\mathrm{C}_{\text {meso }}$ bonds and the direct pyrrole-pyrrole bond (Figure 2). Substituting the 10- $\mathrm{CH}_{2}$ group with divalent groups with a $\pi$ lone pair (i.e., $\mathrm{X}=\mathrm{NH},{ }^{5,29-31} \mathrm{O},{ }^{5,32,33} \mathrm{PH}$, and $\mathrm{S}^{5,33-35}$ ) dramatically boosts the global diatropic ring current, clearly signifying global aromaticity. The results presented in Figure 3 allow for several interesting observations.

To a first approximation, the current density plots for all the aromatic systems are topologically similar in that the great majority of the diatropic current flows along the outer rim of the molecules, with only a smaller amount flowing through the inner $\mathrm{C}_{\alpha}-\mathrm{N}$ bonds. ${ }^{36}$ That said, a couple of significant variations among the different compounds are pointed out. First, nickel complexation results in a modest increase in both the global diatropic current (as measured by that along the $\mathrm{C}_{\alpha}-\mathrm{C}_{\text {meso }}$ bonds) and in the current along the inner 15-membered ring, that is, the $\mathrm{C}_{\alpha}-\mathrm{N}$ bonds. Second, the current along the $\mathrm{C}_{\alpha}-\mathrm{X}$ bonds varies significantly across $\mathrm{X}=\mathrm{NH}, \mathrm{O}, \mathrm{PH}$, and $\mathrm{S}$. Indeed, the global diatropic current exhibits a rough inverse correlation with the electronegativity of X. Presumably, the high electronegativity of oxygen limits its ability to engage in mesomeric electron donation relative to the other $\mathrm{X}$ groups. That said, even for the oxaisocorrole derivatives (Figure 3), the global diatropic current is still considerable, about $30 \%$ higher than that calculated for benzene at the present level of theory $(12.2 \mathrm{nA} / \mathrm{T}){ }^{37}$ Gratifyingly, these theoretical findings are fully consistent with the experimental ones of Bröring and co-workers, who estimated global aromaticity on the basis of the chemical shifts of meso $\mathrm{CH}$ and central $\mathrm{NH}$ hydrogens. ${ }^{5}$

In contrast to the above molecules, the experimentally unprecedented 10-boraisocorrole derivatives $\mathrm{H}_{2}$ [10-B-IsoCor] and $\mathrm{Ni}$ [10-B-IsoCor] (Figure 4) exhibit a high paratropic global current, clearly indicating an antiaromatic macrocycle. For $\mathrm{H}_{2}$ [10-B-IsoCor], the $\mathrm{C}_{\alpha}-\mathrm{C} / \mathrm{X}_{\text {meso }}$ current intensity is approximately $-25 \mathrm{nA} / \mathrm{T}$, whereas for the corresponding nickel complex the analogous current intensity is some $50 \%$ higher, presumably reflecting higher $\pi$-electron mobility because of the increased anionic character of the nitrogens in the metal complex. Importantly, the global current in both of these molecules largely flows along the inner 15 -membered $\mathrm{C}_{11} \mathrm{~N}_{4}$ ring of the macrocycle, a feature that is also shared by other antiaromatic porphyrinoids. ${ }^{38}$ That said, the overall current passes almost exclusively through the inner $\mathrm{C}_{11} \mathrm{~N}_{4}$ ring for the free base, roughly a quarter of the global current along the outer rim for the Ni complex.

Fascinatingly, the present calculations clearly implicate the experimentally known 'oxocorrole ${ }^{39}$ ring system as antiaromatic according to the magnetic criterion (Figure 4). Thus, $\mathrm{H}_{2}[10-$ CO-IsoCor] sustains a global paratropic current of roughly $-10 \mathrm{nA} / \mathrm{T}$, whereas $\mathrm{Ni}$ [10-CO-IsoCor] sustains an even stronger current. A couple of minor twists are worth pointing out. Thus, whereas all four pyrrole rings in $\mathrm{Ni}$ [10-CO-IsoCor] sustain a paratropic current, the two pyrrole rings adjoining the carbonyl group in $\mathrm{H}_{2}$ [10-CO-IsoCor] sustain local diatropic currents. The Ni complex also exhibits a marked asymmetry between the $\mathrm{C}_{9 / 11}-\mathrm{C}_{10}$ bonds and $\mathrm{C}_{1}-\mathrm{C}_{19}$ bond, with a much higher current along the latter.

The finding that both 10-boraisocorrole and oxocorrole behave similarly with respect to their antiaromatic character is, of course, not a great surprise. Like a trivalent boron atom, the carbonyl group in oxocorrole may be thought to formally remove two electrons from the corrole $\pi$-system, resulting in magnetic antiaromaticity. Indeed, both ring systems may be thought to be isoelectronic to norcorrole, ${ }^{40,41}$ the smallest fully conjugated tetrapyrrole, which has been demonstrated to be antiaromatic.

Finally, the current density profiles of the two silaisocorrole ${ }^{42}$ derivatives $\mathrm{H}_{2}$ [10-Si-IsoCor] and $\mathrm{Ni}$ [10-Si-IsoCor] $\left(\mathrm{X}=\mathrm{SiMe}_{2}\right.$, 
Figure 5) do not exhibit a significant global current, either diatropic or paratropic. On the other hand, the pyrrole rings exhibit strong, local diatropic currents.
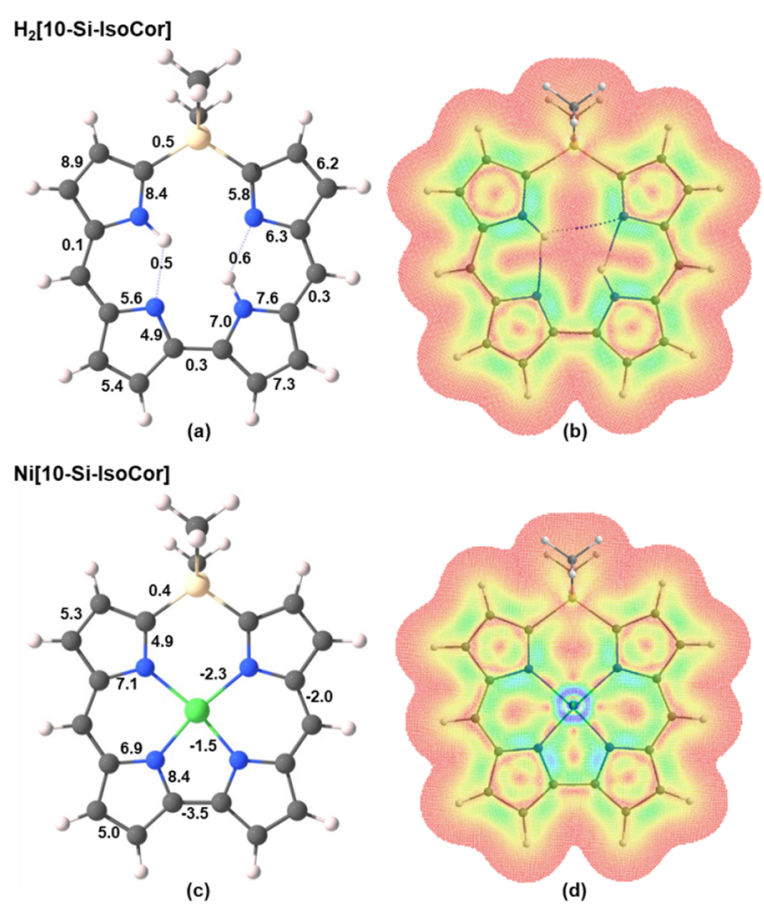

Figure 5. Integrated currents (nA/T, left) and current density plots (right) for $\mathrm{H}_{2}$ [10-Si-IsoCor] and $\mathrm{Ni}[10-\mathrm{Si}$-IsoCor].

\section{CONCLUSIONS}

In summary, DFT calculations have uncovered remarkable magnetic diversity among heteroisocorrole derivatives. Thus, aza-, phospha-, and thiaisocorroles have been found to be strongly aromatic, and oxacorrole is somewhat less, which is nicely consistent with the experimental literature. ${ }^{5}$ Boraisocorrole and oxocorrole, on the other hand, have been found to be strongly antiaromatic, whereas silaisocorrole has been found to be nonaromatic, with respect to a global ring current. Whether heteroisocorrole derivatives exhibit novel photophysical properties and applications remains an exciting question for the future.

\section{METHODS}

All structures were fully optimized at the B3LYP/def2-TZVP computational level using Gaussian 09 rev. $\mathrm{D}^{43}$ and confirmed as local minima by means of frequency analyses. Magnetically induced currents and current density plots were computed by post-analysis of the electron density obtained from GIAO NMR computations using the AIMAll ${ }^{22}$ suite of programs (version 17.01.25). The current magnitudes were obtained within the context of the quantum theory of atoms in molecules developed by Keith and Bader ${ }^{17-21}$ in terms of the integral of the current density passing through the zero-flux surface between two neighboring atoms. The current intensities and plots were all obtained for a magnetic field applied perpendicular to the mean plane of the molecules.

\section{ASSOCIATED CONTENT}

\section{S Supporting Information}

The Supporting Information is available free of charge on the ACS Publications website at DOI: 10.1021/acsomega.8b02626.
Optimized Cartesian coordinates of the molecules studied (PDF)

\section{AUTHOR INFORMATION}

\section{Corresponding Authors}

*E-mail: abhik.ghosh@uit.no (A.G.).

*E-mail: cina.foroutannejad@ceitec.muni.cz (C.F.-N.).

ORCID $\odot$

Cina Foroutan-Nejad: 0000-0003-0755-8173

Abhik Ghosh: 0000-0003-1161-6364

Notes

The authors declare no competing financial interest.

\section{ACKNOWLEDGMENTS}

A.G. acknowledges financial support from the Research Council of Norway (Grant No. 262229). C.F.-N. acknowledges (a) "Projects of Large Research, Development, and Innovations Infrastructures" for access to the computational resources provided by the CESNET LM2015042 and the CERIT Scientific Cloud LM2015085 and (b) project CEITEC 2020 LQ1601 with financial support from the Ministry of Education, Youth, and Sports of the Czech Republic under the National Sustainability Programme II.

\section{REFERENCES}

(1) Pomarico, G.; Xiao, X.; Nardis, S.; Paolesse, R.; Fronczek, F. R.; Smith, K. M.; Fang, Y.; Ou, Z.; Kadish, K. M. Synthesis and Characterization of Free-Base, Copper, and Nickel Isocorroles. Inorg. Chem. 2010, 49, 5766-5774.

(2) Costa, R.; III Geier, G. R.; Ziegler, C. J. Structure and Spectroscopic Characterization of Free Base and Metal Complexes of 5,5-Dimethyl-10,15-Bis(Pentafluorophenyl)Isocorrole. Dalton Trans. 2011, 40, 4384-4386.

(3) Hoffmann, M.; Cordes, B.; Kleeberg, C.; Schweyen, P.; Wolfram, B.; Bröring, M. Template Synthesis of Alkyl-Substituted Metal Isocorroles. Eur. J. Inorg. Chem. 2016, 2016, 3076-3085.

(4) Thomas, K. E.; Beavers, C. M.; Gagnon, K. J.; Ghosh, A. $\beta$ Octabromo- and $\beta$-Octakis(Trifluoromethyl)Isocorroles: New Sterically Constrained Macrocyclic Ligands. ChemistryOpen 2017, 6, 402409.

(5) Sakow, D.; Böker, B.; Brandhorst, K.; Burghaus, O.; Bröring, M. 10-Heterocorroles: Ring-Contracted Porphyrinoids with Fine-Tuned Aromatic and Metal-Binding Properties. Angew. Chem. Int. Ed. 2013, $52,4912-4915$

(6) Umasekhar, B.; Shetti, V. S.; Ravikanth, M. Heterocorroles: Corrole Analogues Containing Heteroatom(s) in the Core or at a Meso-Position. RSC Adv. 2018, 8, 21100-21132.

(7) Fitzgerald, J. P.; Haggerty, B. S.; Rheingold, A. L.; May, L.; Brewer, G. A. Iron Octaethyltetraazaporphyrins: Synthesis, Characterization, Coordination Chemistry, and Comparisons to Related Iron Porphyrins and Phthalocyanines. Inorg. Chem. 1992, 31, 2006-2013.

(8) Ghosh, A. Electronic Structure of Corrole Derivatives: Insights from Molecular Structures, Spectroscopy, Electrochemistry, and Quantum Chemical Calculations. Chem. Rev. 2017, 117, 3798-3881.

(9) Bröring, M.; Köhler, S.; Pietzonka, C. Pseudohalogenido Complexes of Iron-2,2'-Bidipyrrins. J. Porphyr. Phthalocyanines 2012, $16,641-650$.

(10) Sakow, D.; Baabe, D.; Böker, B.; Burghaus, O.; Funk, M.; Kleeberg, C.; Menzel, D.; Pietzonka, C.; Bröring, M. Iron 10Thiacorroles: Bioinspired Iron(III) Complexes with an Intermediate Spin (S=3/2) Ground State. Chem. - Eur. J. 2014, 20, 2913-2924.

(11) Teo, R. D.; Hwang, J. Y.; Termini, J.; Gross, Z.; Gray, H. B. Fighting Cancer with Corroles. Chem. Rev. 2016, 117, 2711-2729. 
(12) Foroutan-Nejad, C.; Larsen, S.; Conradie, J.; Ghosh, A. Isocorroles as Homoaromatic NIR-Absorbing Chromophores: A First Quantum Chemical Study. Sci. Rep. 2018, 8, 11952.

(13) Becke, A. D. Density-Functional Exchange-Energy Approximation with Correct Asymptotic Behavior. Phys. Rev. A 1988, 38, 30983100 .

(14) Lee, C.; Yang, W.; Parr, R. G. Development of the Colle-Salvetti Correlation-Energy Formula into a Functional of the Electron Density. Phys. Rev. B 1988, 37, 785-789.

(15) Miehlich, B.; Savin, A.; Stoll, H.; Preuss, H. Results Obtained with the Correlation Energy Density Functionals of Becke and Lee, Yang and Parr. Chem. Phys. Lett. 1989, 157, 200-206.

(16) Weigend, F.; Ahlrichs, R. Balanced Basis Sets of Split Valence, Triple Zeta Valence and Quadruple Zeta Valence Quality for H to Rn: Design and Assessment of Accuracy. Phys. Chem. Chem. Phys. 2005, 7, 3297-3305.

(17) Keith, T. A.; Bader, R. F. W. Calculation of Magnetic Response Properties Using Atoms in Molecules. Chem. Phys. Lett. 1992, 194, 1-8. (18) Keith, T. A.; Bader, R. F. W. Calculation of Magnetic Response Properties Using a Continuous Set of Gauge Transformations. Chem. Phys. Lett. 1993, 210, 223-231.

(19) Keith, T. A.; Bader, R. F. W. Topological Analysis of Magnetically Induced Molecular Current Distributions. J. Chem. Phys. 1993, 99, $3669-3682$.

(20) Keith, T. A. Calculation of Magnetizabilities Using GIAO Current Density Distributions. Chem. Phys. 1996, 213, 123-132.

(21) Keith, T. A.; Bader, R. F. W. Properties of Atoms in Molecules: Nuclear Magnetic Shielding. Can. J. Chem. 1996, 74, 185-200.

(22) Keith, T. A. AIMAll; Gristmill Software: Overland Park, KS, 2017.

(23) Childs, R. F. The Homotropylium Ion and Homoaromaticity. Acc. Chem. Res. 1984, 17, 347-352.

(24) Gibson, C. M.; Havenith, R. W. A.; Fowler, P. W.; Jenneskens, L. W. Planar Homotropenylium Cation: A Transition State with Reversed Aromaticity. J. Org. Chem. 2015, 80, 1395-1401.

(25) Breslow, R.; Foss, F. W., Jr. Charge Transport in Nanoscale Aromatic and Antiaromatic Systems. J. Phys. Condens. Matter 2008, 20, 374104.

(26) Breslow, R. Antiaromaticity. Acc. Chem. Res. 1973, 6, 393-398.

(27) Fujii, S.; Marqués-González, S.; Shin, J.-Y.; Shinokubo, H.; Masuda, T.; Nishino, T.; Arasu, N. P.; Vázquez, H.; Kiguchi, M. HighlyConducting Molecular Circuits Based on Antiaromaticity. Nat. Commun. 2017, 8, 15984.

(28) Cho, S.; Yoon, Z. S.; Kim, K. S.; Yoon, M.-C.; Cho, D.-G.; Sessler, J. L.; Kim, D. Defining Spectroscopic Features of Heteroannulenic Antiaromatic Porphyrinoids. J. Phys. Chem. Lett. 2010, 1, 895-900.

(29) Johnson, A. W.; Kay, I. T.; Rodrigo, R. 432. 2,2'-Bipyrrolic Macrocyclic Ring Systems. J. Chem. Soc. (Resumed) 1963, 0, 23362342.

(30) Horie, M.; Hayashi, Y.; Yamaguchi, S.; Shinokubo, H. Synthesis of Nickel(II) Azacorroles by Pd-Catalyzed Amination of $\alpha, \mathrm{A}^{\prime}$ Dichlorodipyrrin $\mathrm{Ni}^{\mathrm{II}}$ Complex and Their Properties. Chem. - Eur. J. 2012, 18, 5919-5923.

(31) Omori, H.; Hiroto, S.; Shinokubo, H. The Synthesis of $\mathrm{Ni}^{\mathrm{II}}$ and $\mathrm{Al}^{\mathrm{III}}$ 10-Azacorroles through Coordination-Induced Cyclisation Involving 1,2-Migration. Chem. Commun. 2016, 52, 3540-3543.

(32) Bröring, M.; Brégier, F.; Cónsul Tejero, E.; Hell, C.; Holthausen, M. C. Revisiting the Electronic Ground State of Copper Corroles. Angew. Chem. Int. Ed. 2007, 46, 445-448.

(33) Rösner, J.; Cordes, B.; Bahnmüller, S.; Homolya, G.; Sakow, D.; Schweyen, P.; Wicht, R.; Bröring, M. Heterocorrole Conformations: Little Saddling, Much Ruffling. Angew. Chem. Int. Ed. 2017, 56, 99679970.

(34) Broadhurst, M. J.; Grigg, R.; Johnson, A. W. Sulphur Extrusion Reactions Applied to the Synthesis of Corroles and Related Systems. J. Chem. Soc., Perkin Trans. 1 1972, 0, 1124-1135.

(35) Kamiya, H.; Kondo, T.; Sakida, T.; Yamaguchi, S.; Shinokubo, H. Meso-Thiaporphyrinoids Revisited: Missing of Sulfur by Small Metals. Chem. - Eur. J. 2012, 18, 16129-16135.
(36) Jusélius, J.; Sundholm, D. The Aromatic Pathways of Porphins, Chlorins and Bacteriochlorins. Phys. Chem. Chem. Phys. 2000, 2, 21452151.

(37) Janda, T.; Foroutan-Nejad, C. Why Is Benzene Unique? Screening Magnetic Properties of $\mathrm{C}_{6} \mathrm{H}_{6}$ Isomers. ChemPhysChem 2018, 19, 2357-2363.

(38) Valiev, R. R.; Fliegl, H.; Sundholm, D. Optical and Magnetic Properties of Antiaromatic Porphyrinoids. Phys. Chem. Chem. Phys. 2017, 19, 25979-25988.

(39) Bröring, M.; Hell, C.; Brégier, F.; Burghaus, O.; Cónsul Tejero, E. New Porphyrinoids: Vanadyl(IV) Complexes of Meso-Aryl-Substituted Corrphycene and 10-Oxocorrole Ligands. Inorg. Chem. 2007, 46, $5477-5479$.

(40) Ito, T.; Hayashi, Y.; Shimizu, S.; Shin, J.-Y.; Kobayashi, N.; Shinokubo, H. Gram-Scale Synthesis of Nickel(II) Norcorrole: The Smallest Antiaromatic Porphyrinoid. Angew. Chem. Int. Ed. 2012, 51, $8542-8545$.

(41) Ghosh, A.; Wasbotten, I. H.; Davis, W.; Swarts, J. C. Norcorrole and Dihydronorcorrole: A Predictive Quantum Chemical Study. Eur. J. Inorg. Chem. 2005, 2005, 4479-4485.

(42) Omori, H.; Hiroto, S.; Shinokubo, H. 10-Silacorroles Exhibiting Near-Infrared Absorption and Emission. Chem. - Eur. J. 2017, 23, 7866-7870

(43) Frisch, M. J., ,et al. Gaussian 09; Gaussian, Inc.: Wallingford, CT, 2013. 